\title{
Buoyancy and electrically driven convection models in thin-layer electrodeposition
}

\author{
Guillermo Marshall and Pablo Mocskos \\ Facultad de Ciencias Exactas y Naturales, Universidad de Buenos Aires, Ciudad Universitaria, 1428 Buenos Aires, Argentina
}

Harry L. Swinney and John M. Huth

Center for Nonlinear Dynamics and The Department of Physics, The University of Texas, Austin, Texas 78712

(Received 15 May 1998; revised manuscript received 12 October 1998)

\begin{abstract}
Recent work demonstrates the inevitable role of convection during the growth of patterns in thin-layer electrochemical deposition. Convection is driven mainly by Coulombic forces due to local charges and by buoyant forces due to concentration gradients that lead to density gradients. Here we study by theoretical and numerical modeling the limiting regimes under which electroconvection or gravitoconvection prevail. The model describes the diffusive, migratory, and convective motion of ions in a fluid subject to an electric field. The equations are written in terms of dimensionless quantities, in particular, the gravity Grashof and the electrical Grashof numbers. The simulations reveal that gravitoconvection becomes increasingly important as the gravity Grashof number increases, while electroconvection becomes increasingly important as the electrical Grashof number increases. For both regimes the model predicts concentration, electric potential, and velocity patterns that are in qualitative agreement with typical electrodeposition experiments. In gravitoconvection, the model predicts the evolution, before collision, of the convection rolls near each electrode growing first as $t^{4 / 5}$ and then slowing down to $t^{1 / 2}$; the same scaling behavior was observed in experiments. After collision, the cathodic and anodic rolls merge into a single roll. In electroconvection, the model predicts the existence of vortex pairs formed by the electrical force on space charge accumulating near the growing filament tip. Such vortex rolls and pairs have been observed in experiments. [S1063-651X(99)11102-4]
\end{abstract}

PACS number(s): 81.15.Pq, 82.45.+z, 47.20.Bp, 89.80.+h

\section{INTRODUCTION}

Electrochemical deposition (ECD) of ramified deposits in thin-layer cells, producing complex geometries of fractal or dendritic character [1-5], is a paradigmatic model for studying growth pattern formation. The electrolytic cell consists of two glass plates sandwiching two parallel electrodes and a metal salt electrolyte. A voltage difference applied between electrodes produces a treelike deposit by a reduction of the metal ions. The electrodeposit structure ranges from fractal to dense branch morphology, depending on parameters such as cell geometry, solution concentration, or voltage difference; the morphology variation is not yet fully understood.

Although the existence of convective ion transport has long been recognized [6], its relevance has become appreciated with recent concentration measurements using interferometric, optical absorption, schlieren, and shadowgraph techniques [6-14]. Recent work demonstrated the importance of hydrodynamic motion in electrochemical transport processes in thin-layer cells in the vicinity of the electrodes [14-20]. Fluid motion is mainly produced by electrical and buoyant forces. The importance of electrically induced convection was emphasized in $[15,16]$. In particular, [19] studied the role of induced convection in ECD morphology selection, in the limiting cases of gravitoconvection and electroconvection by means of very precise interferometric concentration measurements, using horizontal and a vertical cell configurations, respectively. In [20] it was concluded that, for ECD in cells with thickness less than $50 \mu \mathrm{m}$, diffusion and migration are the dominant modes in ion transport. In [18], a particle image velocimetry technique was developed that enabled the first direct measurements of convective motion in electrodeposition, demonstrating the importance of buoyancy driven convection. Figure 1(a) is an example of buoyancy driven convection: tracer particles are shown in a vertical plane perpendicular to the electrodes. Figure 1(b) is an example of electrically driven convection: tracer particles are shown in a horizontal plane parallel to the electrodes.

Figure 2 shows a typical cell used in electroconvection experiments. A thin layer of metal salt solution is confined between two parallel glass plates separated by a small gap distance $d$ and bounded at either end by wire electrodes (usually zinc or copper) of length $w$, separated by a distance $l$. Fluid flow is visualized with tracer particles (1-3 $\mu \mathrm{m} \mathrm{di-}$ ameter) of the same density as the electrolytic solution. Figure 1(b) is a top view of an experiment, useful for visualizing electroconvective fluid motion occurring in the quasiplane of the growth. Buoyancy driven flow, however, occurs perpendicular to the quasiplane of growth and is studied by viewing from the side, as in Fig. 1(a).

To explain ECD we need to develop a global macroscopic electro-chemical-fluid dynamical model. In an experiment, when the circuit is closed, current starts flowing through the electrolyte: cations and anions move toward the cathode and anode, respectively [21,22]. Because of ion depletion near the cathode (cation aggregation and anion migration) and ion enhancement near the anode (anions piling up and cations entering through the dissolving anode), in an initial period, that is, before any growth is visible at the cathode, zones rapidly develop with low metal ion concentration (low density) near the cathode and high metal ion concentration (high density) near the anode. This unstable configuration generates a density current flow at both electrodes: at the cathode the fluid ascends towards the top confining plate and at the 

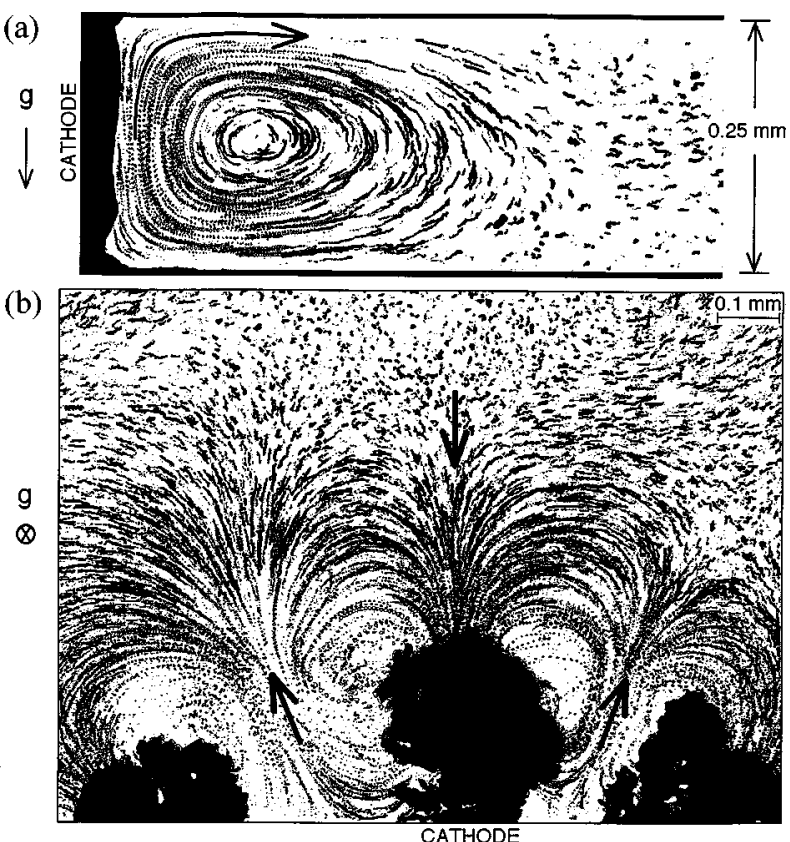

FIG. 1. Convective motion in ECD visualized in an experiment with tracer particles: (a) a side view showing particle trajectories in a vertical plane, for conditions in which gravity convection is dominant, (b) a top view showing particles trajectories in a horizontal plane, for conditions in which electroconvection is dominant (from [18]).

anode the fluid descends towards the bottom. These currents generate a gravity driven vortex tube or roll at each electrode, rotating in the same sense, and expanding toward one another, invading the center of the cell. During this initial period, cation depletion at the cathode is supposed uniform. Simultaneously, in a very narrow region near the cathode a local charge develops, giving rise to Coulombic forces initially pointing towards the cathode.

After a few seconds, an instability develops, triggering the growth of a deposit. The deposit develops as a threedimensional array of thin porous metallic filaments. Coulombic forces concentrate at the tips according to a model developed in [14] (cf. also references therein); each filament allows fluid to penetrate its tip and to be ejected from the sides, forming a toroidal vortex ring driven by the electric force (the three-dimensional vortex rings can form capillary tubes [14]). In the quasiplane of the growth, the threedimensional picture reduces to a pair of counterrotating vor-

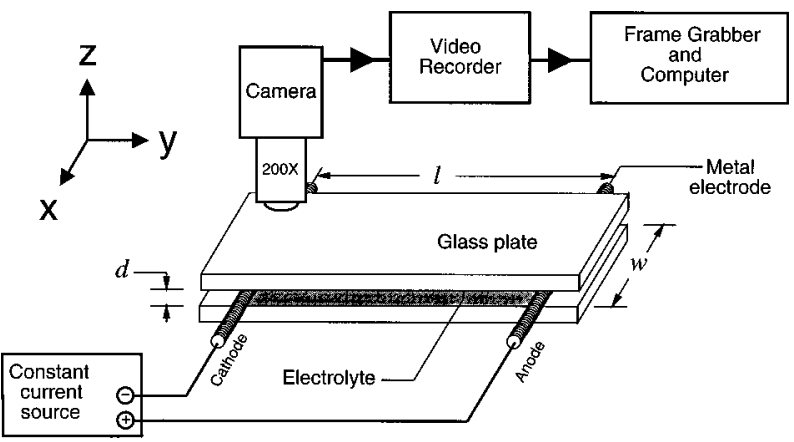

FIG. 2. Schematic diagram of the experimental system (from tices, as described in [14] and illustrated in Fig. 1(b) [18]; in the plane normal to both the quasiplane of the growth and the electrodes, vortex tubes form near the electrodes, as shown in Fig. 1(a). The interaction of electrical driven vortex rings and gravity driven vortex tubes and their effects on the deposit morphology will be discussed in a forthcoming paper.

The morphological structure of the deposit in a given experiment depends on the relative importance of electroconvection and gravitoconvection. Reducing cell thickness and electrolyte concentration is the most direct way for minimizing buoyancy driven convection and enhancing electroconvection $[14,18]$.

To describe particular aspects of the previous scenario, mathematical models in one and two dimensions have been examined [23-28]. In particular, [28] presented a twodimensional model of gravity-induced convection describing a single ion species concentration in a Stokes flow. The interesting results of the model are limited by underlying simplifications including incomplete consideration of the role of diffusive relaxation of the concentration gradients responsible for buoyancy driven convection.

Here we construct a three-dimensional macroscopic model from first principles, including all the relevant physical variables for ion transport: diffusion, migration, and convection driven by Coulombic and buoyant forces and a mechanism that mimics the front growth. We follow ideas developed in [27] and introduce dimensionless numbers governing the dynamics. The three-dimensional model is solved numerically on a lattice, as two bidimensional models, one in a horizontal plane and the other in a vertical plane. The horizontal plane model simulates ECD in the quasiplane of the growth (the plane containing the electrodes); this model describes the limiting case dominated by electroconvection. The vertical plane model simulates ECD in a plane perpendicular to both the quasiplane of the growth and the electrodes; this model describes the limiting case dominated by gravitoconvection. These simulations are in direct analogy with the physical experiments: the horizontal plane model corresponds to top views of the cell where electroconvection limiting regimes are analyzed; the vertical plane model corresponds to side views of the cell where gravitoconvection limiting regimes are analyzed. We conduct a series of simulations of fluid motion to analyze the effects of convection on growth pattern formation. The numerical results are compared with experimental measurements taken from [18].

The plan of the paper is the following. In the next section we examine the physics of ECD, the theoretical model describing it, and a dimensional analysis. The third section is devoted to the computational model. The fourth and fifth sections deal with gravity and electrically driven cases, respectively. The last section draws some general conclusions.

\section{THEORETICAL ANALYSIS}

The physical scenario just described can be studied with a macroscopic mathematical model based on first principles, including the Nernst-Planck equations for transport of ions, the Poisson equation for the electric potential, and the Navier-Stokes equations for the fluid [22]:

$$
\frac{\partial C_{i}}{\partial t}=-\nabla \cdot \mathbf{j}_{i}, \quad i=1,2,
$$




$$
\begin{gathered}
\mathbf{j}_{i}=-\mu_{i} C_{i} \boldsymbol{\nabla} \phi-D_{i} \boldsymbol{\nabla} C_{i}+C_{i} \mathbf{v}, \\
\boldsymbol{\nabla}^{2} \phi=\frac{e}{\epsilon} \sum_{i} z_{i} C_{i}, \\
\frac{\partial \mathbf{v}}{\partial t}+\mathbf{v} \cdot \boldsymbol{\nabla} \mathbf{v}=-\frac{1}{\rho_{0}} \boldsymbol{\nabla} P+\nu \nabla^{2} \mathbf{v}+\frac{\mathbf{f}_{e}}{\rho_{0}}+\frac{\mathbf{f}_{g}}{\rho_{0}}, \\
\boldsymbol{\nabla} \cdot \mathbf{v}=0 .
\end{gathered}
$$

Here $C_{i}, \mathbf{j}_{i}, z_{i}, \mu_{i}$, and $D_{i}$ are, respectively, the concentration, flux, number of charges per ion, mobility and diffusion constants of an ionic species $i ; \phi$ is the electrostatic potential, $e$ is the electronic charge, $\epsilon$ is the permittivity of the medium, $\mathbf{v}$ is the fluid velocity, $P$ is the pressure, $\nu$ is the kinematic viscosity, $\rho_{0}$ is a reference fluid density, and $t$ is the time coordinate. The forcing functions due to Coulombic and buoyant forces are given by

$$
\begin{gathered}
\mathbf{f}_{e}=e \mathbf{E} \sum_{i} z_{i} C_{i}, \\
\mathbf{f}_{g}=+\rho \mathbf{g},
\end{gathered}
$$

where $\mathbf{E}$ is the electric field and $\mathbf{g}$ the gravitational acceleration. Here, for system closure, the following Boussinesq-type approximation is proposed. Assuming a binary mixture ( $i$ $=2$ ) and calling $C_{1}=C$ (cations) and $C_{2}=A$ (anions), the approximation reads

$$
\rho=\rho_{0}(1+\alpha \Delta C+\beta \Delta A)
$$

where

$$
\alpha=+\frac{1}{\rho_{0}} \frac{\partial \rho}{\partial C}, \quad \beta=+\frac{1}{\rho_{0}} \frac{\partial \rho}{\partial A} .
$$

System (1-5), with appropriate initial and boundary conditions, is valid in a space-time domain defined by $\mathbf{G}$ $=[\boldsymbol{\Omega}(\mathbf{t}) \mathbf{x}(\mathbf{0}, \mathbf{t})]$, where $\Omega$ is a three-dimensional region with boundary $\Gamma(t)$; this boundary moves with speed proportional to the norm of the flux $\mathbf{j}_{i}$.

Next we perform a dimensional analysis of system (1-5) written in Cartesian coordinates, introducing the following new variables: $\quad x^{\prime}=x / x_{0} ; y^{\prime}=y / x_{0} ; z^{\prime}=z / x_{0} ; u^{\prime}$ $=u / u_{0} ; v^{\prime}=v / u_{0} ; w^{\prime}=w / u_{0} ; C^{\prime}=C / C_{0} ; A^{\prime}=A / C_{0}$, and $\phi^{\prime}=\phi / \phi_{0}$; the variables with subindices are reference values defined later, and the variables $(u, v, w)$ are the components of the fluid velocity vector. Now we write the system in transport-vorticity form by taking the curl of the Navier-Stokes equations and introducing the vorticity and stream vector functions, thus eliminating the pressure. As previously discussed, we consider the two limiting cases in which either electroconvection or gravitoconvection dominates. They yield the top view and side view models, which we give in terms of dimensionless numbers $M, \mathrm{Pe}, \mathrm{Re}, \mathrm{Po}$, $\mathrm{Ge}$, and $\mathrm{Gg}$ [defined after Eq. (25)]:

Case (a). Electroconvection dominated regime (top view model):

$$
\begin{gathered}
\frac{\partial C}{\partial t}=\frac{1}{\operatorname{Pe}_{C}} \nabla^{2} C+\frac{1}{M_{C}}\left[\frac{\partial}{\partial x}\left(C \frac{\partial \phi}{\partial x}\right)+\frac{\partial}{\partial y}\left(C \frac{\partial \phi}{\partial y}\right)\right] \\
-\frac{\partial}{\partial x}(u C)-\frac{\partial}{\partial y}(v C) \\
\frac{\partial A}{\partial t}=\frac{1}{\mathrm{Pe}_{A}} \nabla^{2} A-\frac{1}{M_{A}}\left[\frac{\partial}{\partial x}\left(A \frac{\partial \phi}{\partial x}\right)+\frac{\partial}{\partial y}\left(A \frac{\partial \phi}{\partial y}\right)\right] \\
-\frac{\partial}{\partial x}(u A)-\frac{\partial}{\partial y}(v A) \\
\frac{\partial \omega}{\partial t}+u \frac{\partial \omega}{\partial x}+v \frac{\partial \omega}{\partial y}=\frac{1}{\operatorname{Re}^{2}} \nabla^{2} \omega+\frac{1}{\operatorname{Po}_{C}} A, \\
+\mathrm{Ge}_{C}\left(\frac{\partial A}{\partial y} \frac{\partial \phi}{\partial y}-\frac{\partial A}{\partial y} \frac{\partial \phi}{\partial x}\right) \\
\operatorname{Po}^{2} \psi=-\omega
\end{gathered}
$$

where $\omega$ represents the vorticity in the horizontal plane and $\psi$ the stream function. The boundary conditions for $(x, y)$ on the cathode and the deposit are

$$
\begin{gathered}
\phi(x, y)=-\frac{k T}{z_{C} e \phi_{0}} \ln \left[z_{C} C(x, y)\right], \\
\frac{\partial C}{\partial n}(x, y)=0,
\end{gathered}
$$

$$
j_{A n}(x, y)=0
$$

where $j_{A n}(x, y)$ is the anion flux, $n$ is the normal to the boundary, $k$ is the Boltzmann constant, and $T$ is the absolute temperature. The boundary conditions for $(x, y)$ on the anode are

$$
\begin{gathered}
\phi(x, y)=1-\frac{k T}{z_{C} e \phi_{0}} \ln \left[z_{C} C(x, y)\right] \\
C(x, y)=A(x, y) \\
j_{A n}(x, y)=0 .
\end{gathered}
$$

The boundary conditions for $(x, y)$ on the lateral sides are $\partial C / \partial n=\partial A / \partial n=0, \partial \phi / \partial n=0$. On every solid boundary the stream function satisfies: $\psi=\partial \psi / \partial n=0$.

Case (b). Gravitoconvection dominated regime (side view model)

$$
\begin{aligned}
\frac{\partial C}{\partial t}= & \frac{1}{\operatorname{Pe}_{C}} \nabla^{2} C+\frac{1}{M_{C}}\left[\frac{\partial}{\partial y}\left(C \frac{\partial \phi}{\partial y}\right)+\frac{\partial}{\partial z}\left(C \frac{\partial \phi}{\partial z}\right)\right] \\
& -\frac{\partial}{\partial y}(v C)-\frac{\partial}{\partial z}(w C),
\end{aligned}
$$


TABLE I. Typical values of dimensionless numbers corresponding to the experiment shown in Fig. 1.

\begin{tabular}{lcccccccc}
\hline \hline Case & $M_{A}$ & $M_{C}$ & Po & Pe & Re & Gg & Ge & $\lambda$ \\
\hline Fig. 1(a) & 1.6 & 2.4 & $6.8 \times 10^{-11}$ & 5 & $2.5 \times 10^{-3}$ & $80 \times 10^{4}$ & $73 \times 10^{11}$ & $11 \times 10^{-8}$ \\
Fig. 1(b) & 0.8 & 1.2 & $6.8 \times 10^{-9}$ & 2 & $1.0 \times 10^{-3}$ & $1.6 \times 10^{4}$ & $2.9 \times 10^{11}$ & $5.5 \times 10^{-8}$ \\
\hline \hline
\end{tabular}

$$
\begin{gathered}
\frac{\partial A}{\partial t}=\frac{1}{\mathrm{Pe}_{A}} \nabla^{2} A-\frac{1}{M_{A}}\left[\frac{\partial}{\partial y}\left(A \frac{\partial \phi}{\partial y}\right)+\frac{\partial}{\partial z}\left(A \frac{\partial \phi}{\partial z}\right)\right]-\frac{\partial}{\partial y}(v A) \\
-\frac{\partial}{\partial z}(w A), \\
\nabla^{2} \phi=-\frac{1}{\mathrm{Po}_{C}} C+\frac{1}{\mathrm{Po}_{A}} A \\
\frac{\partial \omega}{\partial t}+v \frac{\partial \omega}{\partial y}+w \frac{\partial \omega}{\partial z}=\frac{1}{\operatorname{Re}} \nabla^{2} \omega-\mathrm{Gg}_{C} \frac{\partial C}{\partial y}-G g_{A} \frac{\partial A}{\partial y} \\
\nabla^{2} \psi=-\omega
\end{gathered}
$$

where $\omega$ represents the vorticity in the vertical plane. The boundary conditions are similar to those on the first case, but without deposit growth. The dimensionless numbers [29] are migration number,

$$
M_{C}=\frac{x_{0} u_{0}}{\mu_{C} \phi_{0}}, \quad M_{A}=\frac{x_{0} u_{0}}{\mu_{A} \phi_{0}} ;
$$

Peclet number,

$$
\mathrm{Pe}_{C}=\frac{x_{0} u_{0}}{D_{C}}, \quad \mathrm{Pe}_{A}=\frac{x_{0} u_{0}}{D_{A}} ;
$$

Poisson number,

$$
\mathrm{Po}_{C}=\frac{\epsilon \phi_{0}}{x_{0}^{2} C_{0} z_{C} e}, \quad \mathrm{Po}_{A}=\frac{\epsilon \phi_{0}}{x_{0}^{2} C_{0} z_{A} e} ;
$$

Reynolds number,

$$
\operatorname{Re}=\frac{x_{0} u_{0}}{\nu} ;
$$

electrical Grashof number

$$
\mathrm{Ge}_{C}=\frac{e z_{C} C_{0} \phi_{0}}{\rho_{0} u_{0}^{2}}, \quad \mathrm{Ge}_{A}=\frac{e z_{A} C_{0} \phi_{0}}{\rho_{0} u_{0}^{2}} ;
$$

gravity Grashof number,

$$
\mathrm{Gg}_{C}=\frac{x_{0} C_{0} g \alpha}{u_{0}^{2}}, \quad \mathrm{Gg}_{A}=\frac{x_{0} C_{0} g \beta}{u_{0}^{2}} .
$$

To calculate the values of these dimensionless numbers from experimental parameters, we associate the typical length scale $x_{0}$ with the gap distance $d$, and estimate the characteristic potential drop over this length scale, $\phi_{0} / d$, by an Ohmic approximation. For a potentiostatic experiment in a rectangular geometry, $\phi_{0}=V d / L$ where $V$ is the experimentally controlled potential across the cell length, $L$. For a galvanostatic experiment with controlled current, $I$, in a rectangular geometry, the typical potential can be estimated by $\phi_{0}=j d /\left[C_{0} e z\left(\mu_{C}+\mu_{A}\right)\right]$, where $j=I / w d$ is the average current density through the cell cross section.

Figure 1(a) depicts a typical ECD experiment in which gravitoconvection is dominant while Fig. 1(b) illustrates the dominance of electroconvection. The experimentally controlled parameters for these experiments were

Fig. 1(a): $d=0.025 \mathrm{~cm}, j=40 \mathrm{~mA} / \mathrm{cm}^{2}, L=1.7 \mathrm{~cm}$, $C_{0}=0.2 \mathrm{M}$,

Fig. 1(b): $d=0.010 \mathrm{~cm}, j=4 \mathrm{~mA} / \mathrm{cm}^{2}, \quad L=2.5 \mathrm{~cm}$, $C_{0}=0.01 M$,

Table I presents typical values of dimensionless numbers corresponding to these experiments. As reference values we use $d$ for $x_{0}, 10 \mu \mathrm{m} / \mathrm{s}$ for $u_{0}$, and $\phi_{0}$ is estimated at $19 \mathrm{mV}$ for case (a) and $15 \mathrm{mV}$ for case (b).

The different values of the dimensionless numbers in Table I reflect the disparity of scales of the processes involved in ECD. The ratio $\lambda=\mathrm{Gg} / \mathrm{Ge}=\rho_{0} g \alpha x_{0} /\left(e z \phi_{0}\right)$ expresses the relative importance of gravitoconvection as compared to electroconvection for a typical ECD experiment. The value of $\lambda$ for the experiment of Fig. 1(a) is twice that of the experiment of Fig. 1(b), consistent with the observation of greater relative influence of gravitoconvection in the experiment of Fig. 1(a).

By grouping the original variables in a particular combination we have reduced the large number of physicochemical parameters involved in ECD to a few dimensionless numbers. Inspection of the equations governing both regimes shows that they share the Pe, $M$, Po, and Re numbers, but the dimensionless numbers acting on the Navier-Stokes equations differ for the two regimes. When electroconvection prevails, the Ge number becomes important, convection increasing with it; when gravitoconvection dominates the picture, the Gg number becomes important, convection increasing with it.

We now comment on the values of the dimensionless numbers. The aim of a numerical simulation is to describe reality as close as possible; hence the simulations should use the same values for the dimensionless numbers as used in the experiments. However, this is not possible in our study because of computer limitations and numerical stability problems. We are obliged to use different numbers, thus simulating a different physical problem, but hopefully one with close resemblance to reality. Thus the aim of our numerical simulation is not a quantitative comparison, but to show that the patterns obtained are qualitatively similar to those observed in experiment. In fact, the correspondence is surprisingly good, indicating that the model captures the most relevant physical variables describing ECD. 
(a)
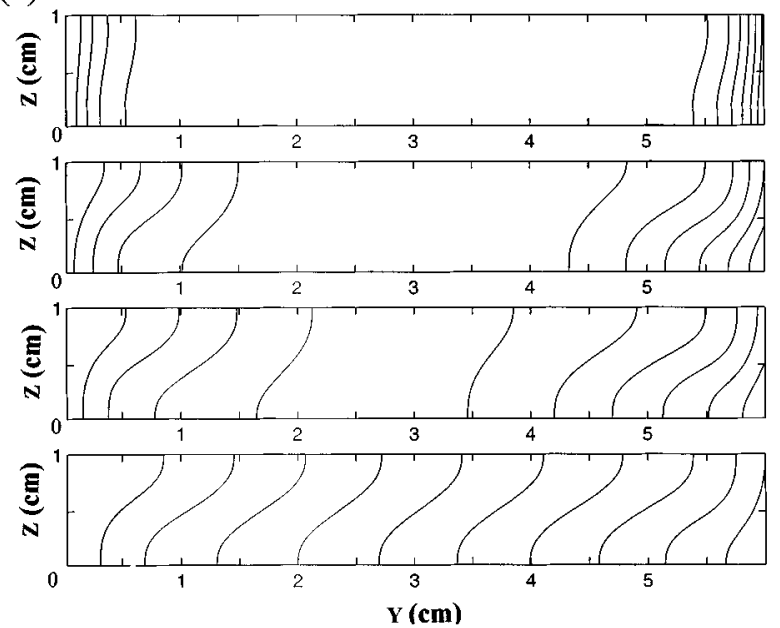

(b)

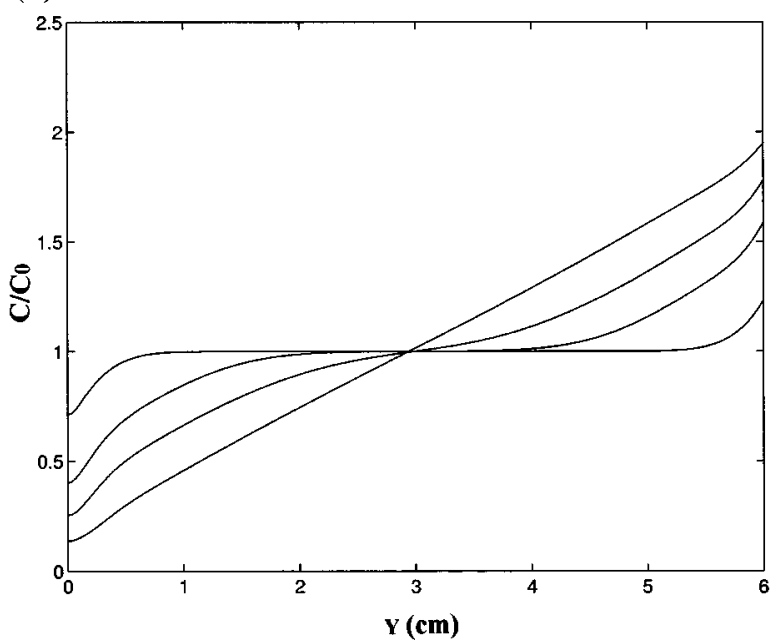

(c)
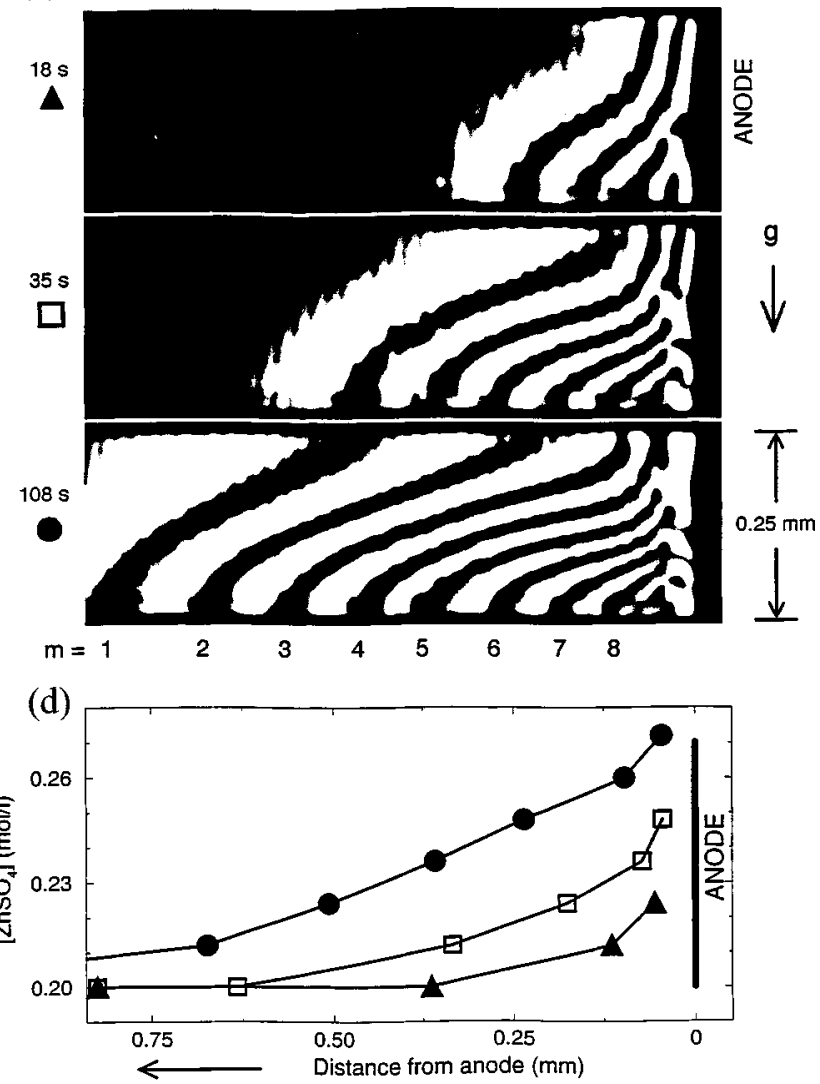

FIG. 3. (a) Side view model simulation (the cell is $6 \times 1 \mathrm{~cm}^{2}$ ) of cation concentration contour lines for $\mathrm{Gg}=10$ and $\mathrm{t}=10,50,100$, and 215 seconds (time increasing downwards), (b) cation concentrations at mid-depth $(z=0.5 \mathrm{~cm})$ as a function of distance between electrodes, (c) interferrometric experimental measurement showing lines of constant density near the anode at different times (from [18]), (d) experimental ion concentration at mid-depth as a function of distance from anode (from [18]).

\section{COMPUTATIONAL MODEL}

The three-dimensional model is treated as a set of two bidimensional models: the horizontal plane (top view) model and the vertical plane (side view) model. These models simulate the limiting cases of electroconvection or gravitoconvection, respectively.

The computational model solves system (10-14) or system $(21-25)$, for each time step, in a fixed domain, in a two-dimensional uniform lattice (equal spacing in both directions) using finite differences and deterministic relaxation techniques. Its solution is obtained via the system of difference equations:

$$
\mathbf{W}_{k}^{n+1}=\sum_{j} \mathbf{a}_{j} \mathbf{W}_{j}^{n},
$$

where $j$ represents the nearest-neighbor site of the site $k$; the summation ranges over all nearest-neighbor sites; $\mathbf{W}_{k}$ is a vector-valued function, the components are the concentrations $C$ and $A$, the electrostatic potential $\phi$, the vorticity function $\omega$, and the stream function $\psi$; and $\mathbf{a}_{j}$ is a diagonal matrix the elements of which contain the nonlinear coefficients of the discretized equations. The resulting solution $\mathbf{W}_{k}^{n+1}$ is then used to advance the interface with a dielectrical breakdown model (DBM) [27]. The interface is moved at random, proportionate to the flux of cations, i.e.,

$$
p_{k}=\frac{\left|\mathbf{j}_{c k}\right|}{\sum_{i}\left|\mathbf{j}_{c i}\right|},
$$

where $k$ is a nearest-neighbor site to the interface, $p_{k}$ is the probability of selecting the nearest-neighbor site $k$ to advance the interface, the summation is over all nearestneighbor sites $i$ to the interface, and $\mathbf{j}_{c k}$ is the flux of cations flowing from the neighbor site $k$ into the aggregation.

\section{GRAVITY DRIVEN CONVECTION}

We now present numerical results obtained for the side view case, as in Fig. 1(a) [18]. The experimental cells have an average height-to-length ratio of $R=0.014$, while in the 
simulation a typical value is much larger, $R=0.166$, which is represented in the simulation by a $30 \times 180$ lattice; the computational cell is the largest that we can handle with our present computer resources.

The simulations describe a growthless evolutionary process until the steady state is attained. The dimensionless numbers used are $\mathrm{Pe}=10^{2}, M_{C}=19, M_{A}=12, \mathrm{Po}=4$ $\times 10^{-2}, \operatorname{Re}=1$ and $\mathrm{Gg}=1,5,10$, and 15. Because of numerical constraints, the values of $R$ and the dimensionless numbers differ considerably from the typical experimental values.

Figure 3(a) presents contour lines of cation concentration at early and late times, and Fig. 3(b) shows the corresponding concentrations at mid-depth as a function of the distance between electrodes. The concentration contour lines are not parallel because of the coupling with the other transport processes: migration and convection. As will be shown later, the appearance of two vortex rolls at an early time introduces a torque into the otherwise smooth diffusion process, dramatically changing the concentration pattern, as shown in Fig. 3(a). For comparison, Fig. 3(c) shows interferometric measurements of constant density lines near the anode at early times, and Fig. 3(d) shows the corresponding ionic concentration at mid-depth as a function of distance from the electrode surface. The simulations are in strikingly good qualitative accord with experiment; gradients quickly form [cf. Figs. 3(a) and 3(c)], despite the difference in the values of the dimensionless parameters.

The gradients that form at mid-depth in the simulations [Fig. 3(b)] are qualitatively similar to those in the experiment [Fig. 3(d)], even though the aspect ratios are quite different ( $R=0.166$ and 0.014 , respectively). One consequence of the different aspect ratios is that the simulated front speed is larger than in the experiments. There are also quantitative differences because of the difference in the values of the dimensionless numbers; for example, the concentration gradients in the simulations are smaller than those observed in experiments.

Figure 4 shows simulated velocity components at an early time: $V_{z}$ along the cell, $V_{y}$ as a function of height near the cathode, and $V_{y}$ as function of height near the anode, respectively. The simulated curves are quite similar to experimental observations-compare in [18] Figs. 4(a)-(d), 4(f), and 4(e), respectively. Both experiment and simulation reveal the formation at early times of vortex rolls near the cathode and anode, respectively.

Figure 5(a) presents stream function contours for different times, showing the existence and space-time evolution of cathode and anode vortex rolls. The region entrained by the buoyancy-driven convection grows with time, invading the cell from both ends. Between the rolls there is no motion until the rolls grow and collide. After the collision, the fluid is entrained in a single long vortex. The sequence in Fig. 5(a) shows that the anode vortex is larger than the cathode one, suggesting that buoyancy forces are larger at the anode, which is in accord with velocity simulations. An experimentally observed sequence of pictures of trace particles [Fig. 5(b)] shows the same behavior as the simulation-near the cathode the depleted electrolyte rises and drives a clockwise flow, and near the anode the electrolyte with high concentration falls and drives a clockwise flow. (a)

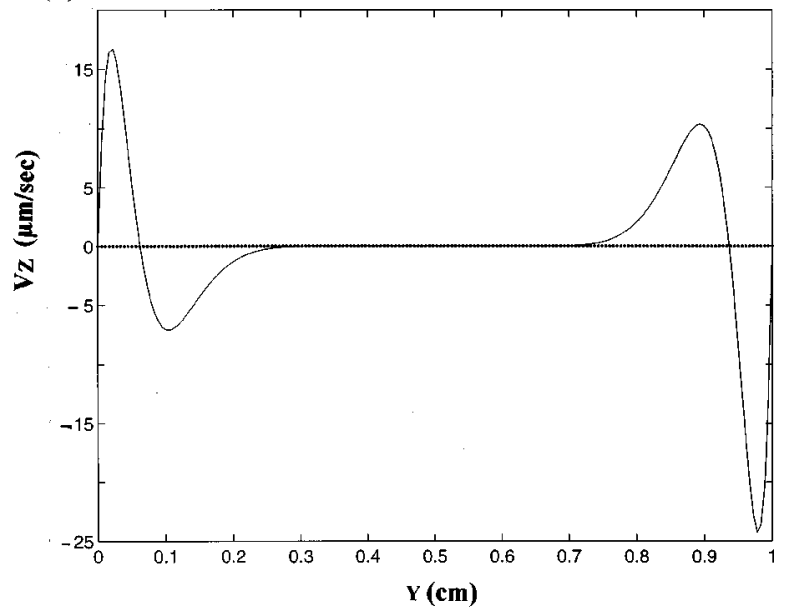

(b)

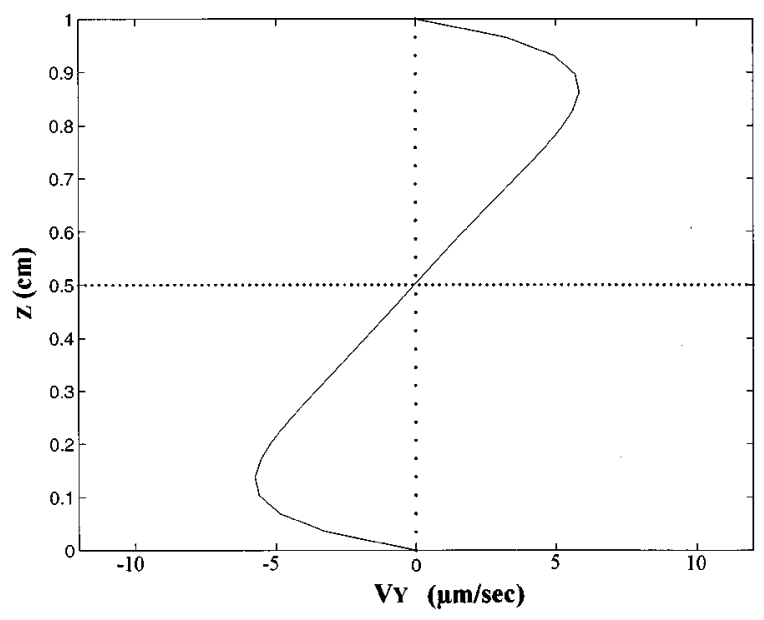

(c)

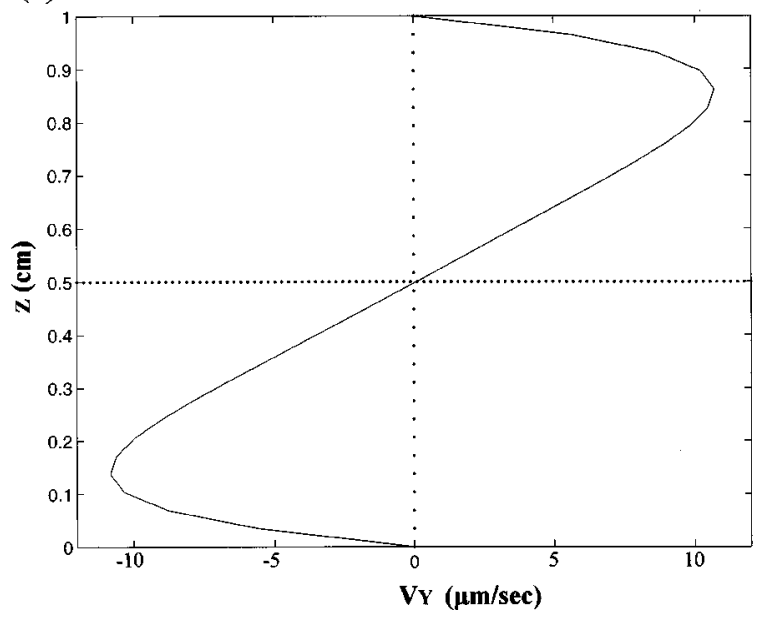

FIG. 4. Side view model simulation of velocity components at $t=5 \mathrm{sec}$ for $\mathrm{Gg}=10$ (the cell is $6 \times 1 \mathrm{~cm}^{2}$ ): (a) Velocity component $V_{z}$ as a function of distance between electrodes, (b) velocity component $V_{y}$ near the cathode (at $y=0.3 \mathrm{~cm}$ ) as a function of height, (c) velocity component $V_{y}$ near the anode (at $y$ $=0.97 \mathrm{~cm}$ ) as a function of height.

To characterize the evolution of the cathodic and anodic convective disturbances, the size of each vortex can be determined as a function of time. The size can be obtained numerically as follows: in each of the stream function con- 
(a)
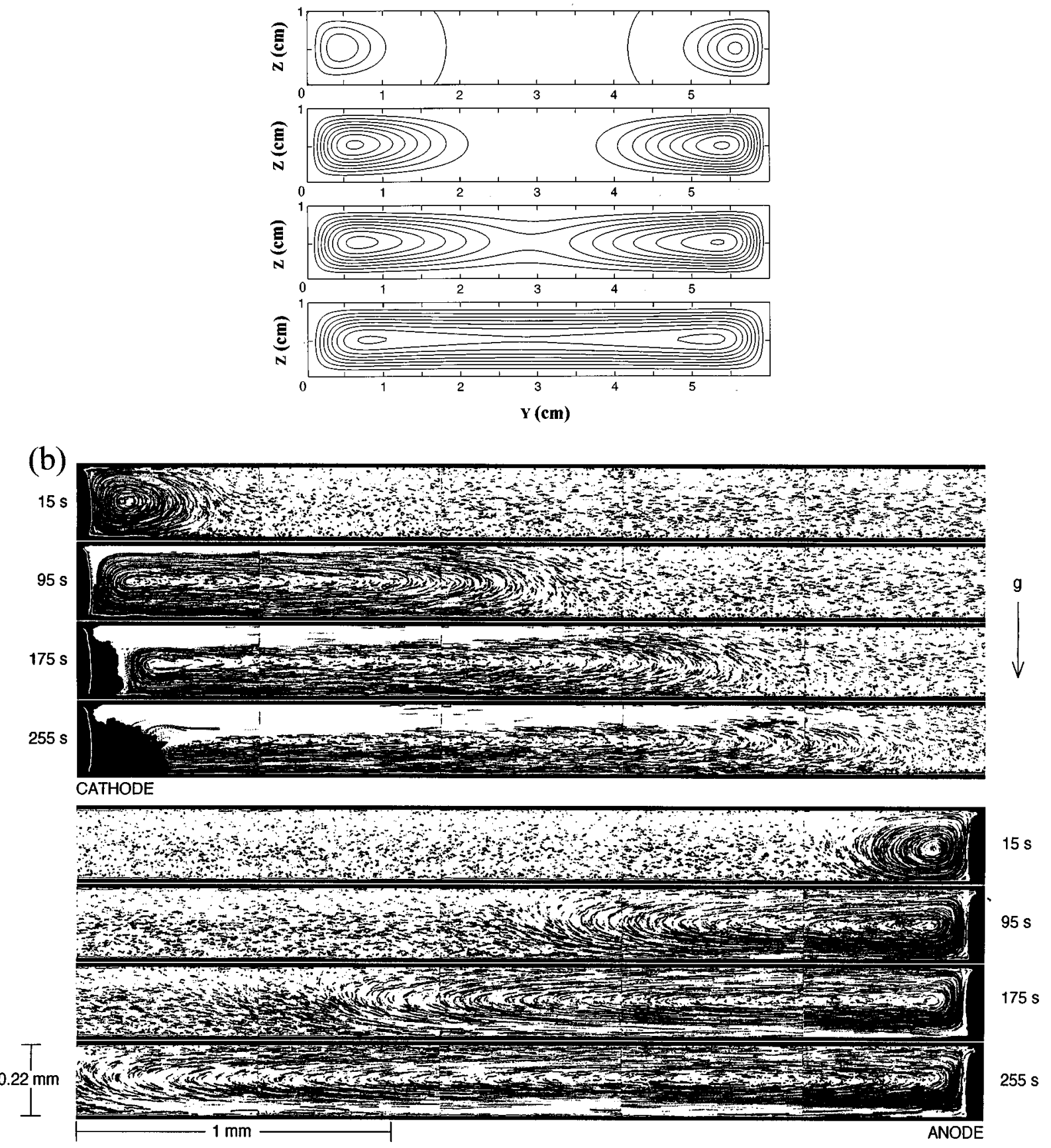

FIG. 5. (a) Side view model simulation (the cell is $6 \times 1 \mathrm{~cm}^{2}$ ) of the stream function contour lines for $\mathrm{Gg}=10 \mathrm{and} \mathrm{t}=10$, 50, 100, and $215 \mathrm{sec}$ (time increasing downwards) showing the cathode and anode vortex evolution before and after collision, (b) side view of an experimental ECD showing the growth of convection rolls, near the cathode where the depleted electrolyte rises and drives a clockwise flow and near the anode where the electrolyte with high concentration falls and also drives a clockwise flow (from [18]).

tour frames of Fig. 5(a), a longitudinal cross section through the vortex centers is computed; a time stack formed from them results in a space-time surface $\psi(y, t)$, the contours of which are plotted in Fig. 6(a). This figure shows the growth of the vortices near the cathode and anode, until the vortices collide and merge. The process continues until a steady state is reached, where contour lines are parallel to the time axis. At that stage, a single vortex occupies the whole cell (not shown in the figure). Figure 6(b) shows the anodic vortex roll size before collision, taken from a particular contour of Fig. 6(a). Figure 6(c) shows the logarithmic plot of the vor- tex roll size of Fig. 6(b). These roll sizes were constructed assuming no deposit growth; although this does not affect the anode roll front, it does affect the cathode roll front, which is slaved to the deposit front.

Experiments have revealed a transition from a regime when the convection rolls grow as $t^{4 / 5}$ at early times to a regime with the roll size growing as $t^{1 / 2}$ for long times as shown in Fig. 6(d). Remarkably, our simulations indicate the same two scaling regimes, as Fig. 6(c) illustrates.

Earlier discussions showed that when gravitoconvection prevails, $\mathrm{Gg}$ is the main dimensionless number governing 
(a)

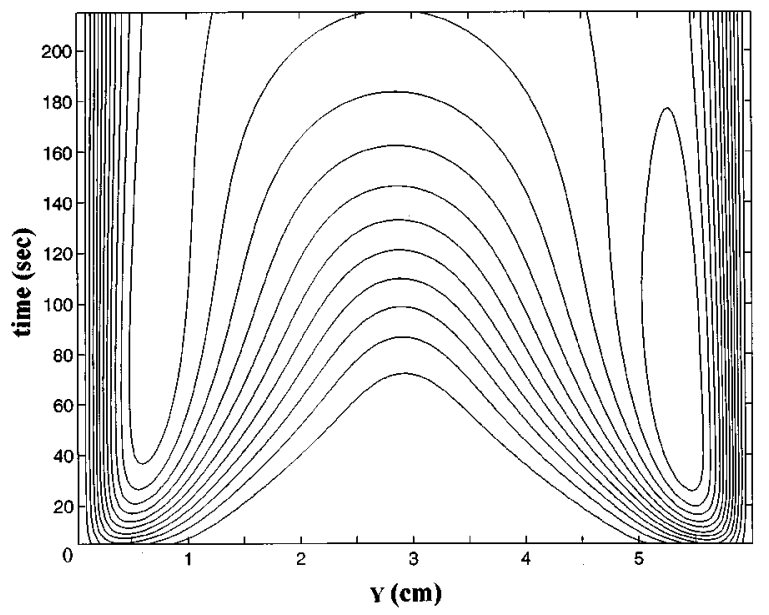

(b)

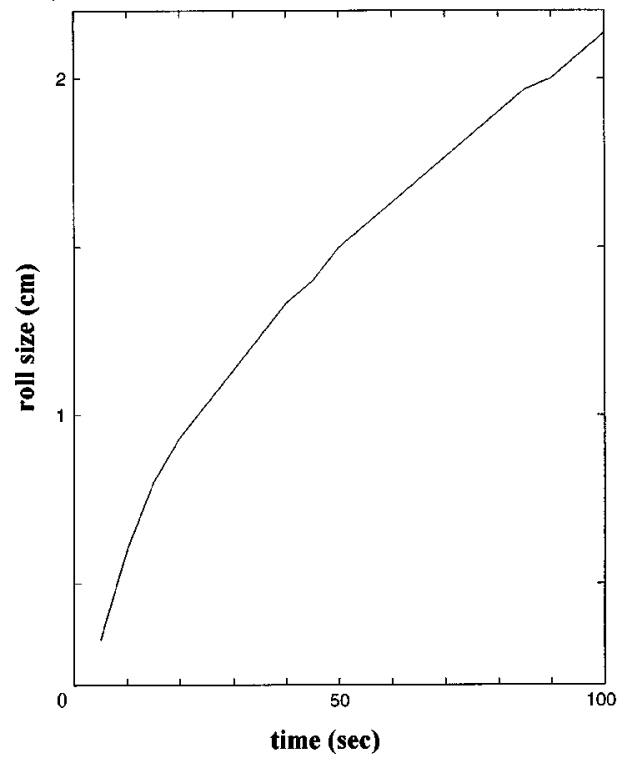

(c)

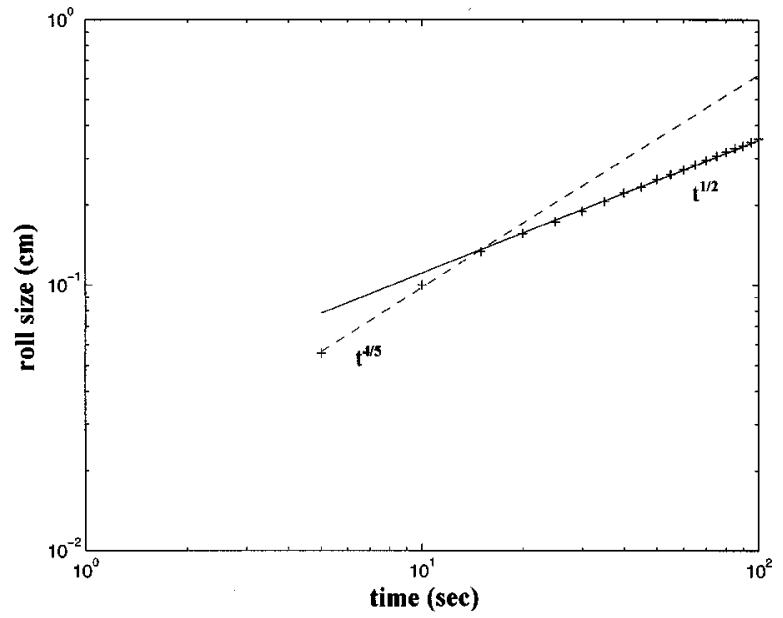

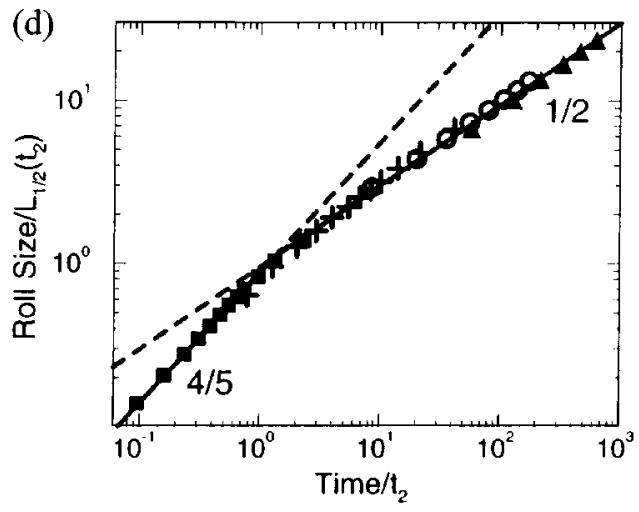

(e)

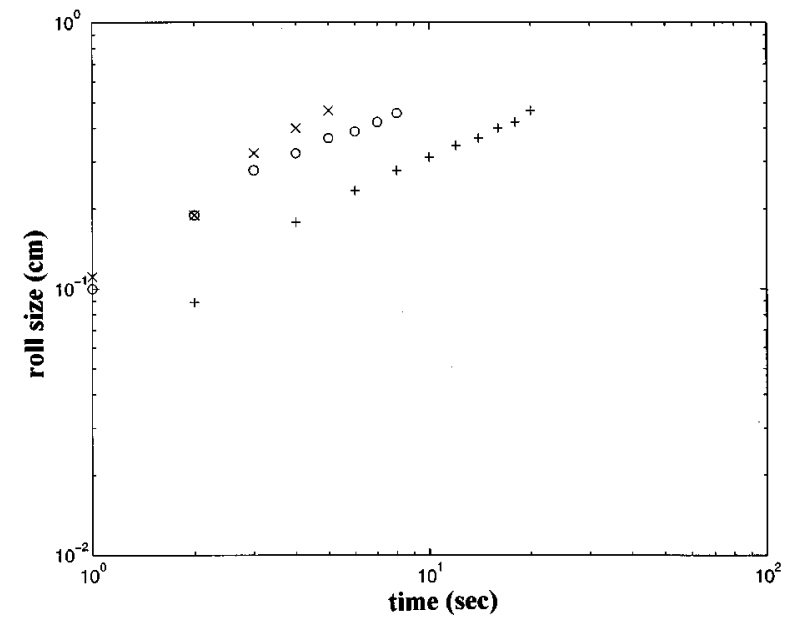

FIG. 6. (a) Side view model simulation (the cell is $6 \times 1 \mathrm{~cm}^{2}$ ) of the contours of the space-time surface $\psi(y, t)$ showing the evolution of cathode and anode vortex motions, in particular, their spatial growth until collision and merging. (b) Simulated roll size before collision. (c) Logarithmic plot of the roll size of Fig. 6(b) (dashed line indicates fit with $t^{4 / 5}$, solid line indicates fit with $t^{1 / 2}$ ). (d) Experimental roll size as a function of time showing the transition from $t^{4 / 5}$ growth to $t^{1 / 2}$ growth. The phenomenological rescaling parameters $L_{1 / 2}$ and $t_{2}$ are discussed in [18]. (e) Simulated roll size as a function of time for different aspect ratios $R$ and $\operatorname{Gg}=10[R=3 / 9 \quad(+), R=4 / 9(\bigcirc)$, and $R=5 / 9(\times)]$. 

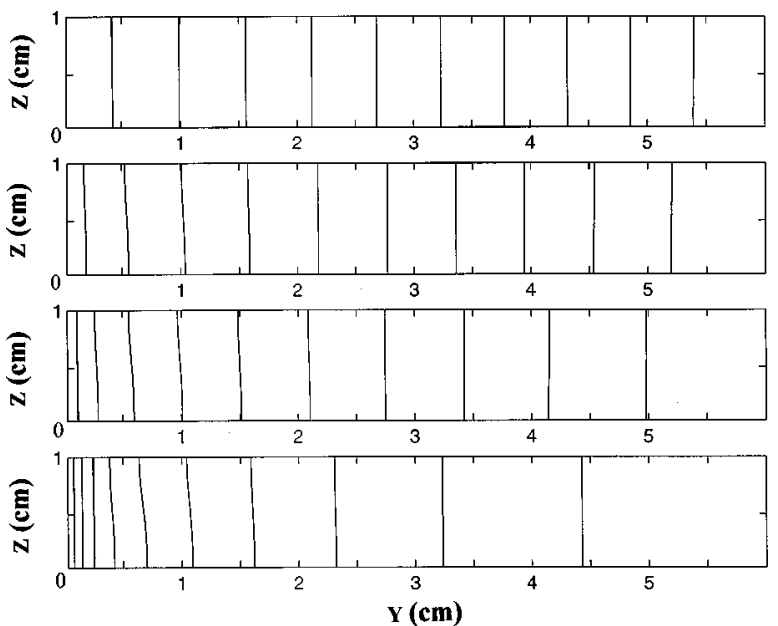

FIG. 7. Side view model simulation (the cell is $6 \times 1 \mathrm{~cm}^{2}$ ) of the electric potential contour lines for $\mathrm{Gg}=10$ and $\mathrm{t}=10,50,100$ and 215 seconds (time increasing downwards).

ECD and that gravitoconvection increases with it. Gg is a function of height, if all other parameters are kept constant, i.e., $\mathrm{Gg}=f($ height $)=f($ length $\times R)$. Hence, $\mathrm{Gg}$ increases with $R$. Figure 6(e) shows the anode roll size as a function of time for different $R$ (here the cell length is kept constant) and constant $\mathrm{Gg}$ - the roll size grows faster for increasing $R$. The general trend of each curve is similar, with the roll size growing at a faster rate during early times (e.g., $t^{4 / 5}$ ) but at a slower rate for long times (e.g., $t^{1 / 2}$ ). Figure 7 presents electric potential contour lines for different times, showing that the electric field grows larger in a narrow zone near the cathode, while the electric field reduces in strength near the anode. This behavior is consistent with the macroscopic changes in electrolyte conductivity that occur as the concentration field evolves [see Fig. 3(b)]. Figure 7 also shows that the electric field varies slightly in the vertical direction. This is apparent from the more tightly compressed equipotential lines (higher field) found in the vicinity of the lower density, lower conductivity plume rising from the cathode surface. Near the anode, the higher density, higher conductivity plume descends, lowering the electric field near the bottom of the cell. Near both electrodes, the electric field is slightly larger in the top half of the cell than in the bottom half. This behavior is consistent with the local changes in electrolyte conductivity that occur as the concentration gradients drive the convective rolls [see Fig. 3(a)]. This vertical variation of the electric field is small, however, suggesting that the electric field is not strongly influenced by convective motion and indicating its leading role in ECD processes (in the absence of ramified growth).

In ending this section we remark that the striking agreement between our model predictions and observations indicates that the set of variables chosen is the most relevant for the description of gravity driven convection.

\section{ELECTRICAL DRIVEN CONVECTION}

We now present numerical results obtained with the top view (horizontal) model, which mimics the conditions in Fig. 1(b). We represent a region near a growing tip by a 150 $\times 150$ uniform lattice; the simulation describes the evolutionary process of growth and electrohydrodynamic interaction. The dimensionless numbers used are: $\mathrm{Pe}_{C}\left(\mathrm{Pe}_{A}\right)$ $=100, M_{C}=19, M_{A}=12, \operatorname{Po}_{C}\left(\mathrm{Po}_{A}\right)=4 \times 10^{-2}$, and $\mathrm{Ge}_{C}$ $\left(\mathrm{Ge}_{A}\right)=10^{3}$ (we assumed $x_{0}=1 \mathrm{~cm}$ and $u_{0}=100 \mu \mathrm{m} / \mathrm{s}$ ). Again, these numbers [except for the $M_{C}\left(M_{A}\right)$ ] are the maximum attainable due to numerical stability constrains.

Figure 8(a) shows the convective zone around a tip growth, Fig. 8(b) the graph of the $V_{y}$ velocity component as a function of the distance to the tip along a vertical line passing through the tip, Fig. 8(c) the graph of the $V_{x}$ velocity component along a horizontal line close to the tip, and Fig. 8(d) the graph of the $V_{y}$ velocity component along a horizontal line close to the tip. These results show the existence of vortex pairs formed by the electrical force on space charge accumulating near the growing filament tip, pulling ions towards the cathode and dragging fluid along. For comparison, Figs. 12 and 13 from [18] show the experimental convective motion near the tip of the aggregation driven mainly by electric forces and the corresponding $V_{x}$ and $V_{y}$ velocity components. In spite of the obvious coarseness of the mesh and different dimensionless numbers used, numerical and physical experiments exhibit a similar trend in vortex pattern and velocity distribution. More extensive results [27] showed the convective zone near the tips of the aggregation as a function of time for different Ge numbers and fixed geometry. Those simulations represented different fluids (the kinematic viscosity was varied), and as expected the velocity increased when the Ge number was increased. The concentration, potential and velocity patterns obtained were in agreement with typical electrodeposition experiments.

\section{DISCUSSION}

We have presented a macroscopic model of electrochemical deposition in the presence of migration, diffusion, and convection. The model has been solved in limiting regimes where buoyancy or electroconvection forces prevail. Of course, in reality the effects of buoyant and electrical forces cannot be considered separately. Buoyant forces are activated by electrical forces which drive the reactions that lead to density variations. In this sense we can say that the main forcing function in ECD is the electrical field. Without it no concentration gradients arise and gravity cannot act. On the other hand, for long times after the electric field is turned off, gravity driven convection persists as the concentration gradients relax.

The two limits that we have considered are oversimplifications, but the reduction of the three-dimensional problem to two two-dimensional problems makes the computations tractable. Even in two dimensions we cannot, because of computational constraints, simulate the behavior for the same parameter values as in the experiments - the values of the dimensionless parameters in the simulations differ significantly from those in the experiments. Nevertheless, despite the differences between the model and the experiments, the simulations capture the essential physical phenomena, as the graphs of concentration, potential, and velocity illustrate. Even the scaling behavior of the growth of the size of the 
(a)

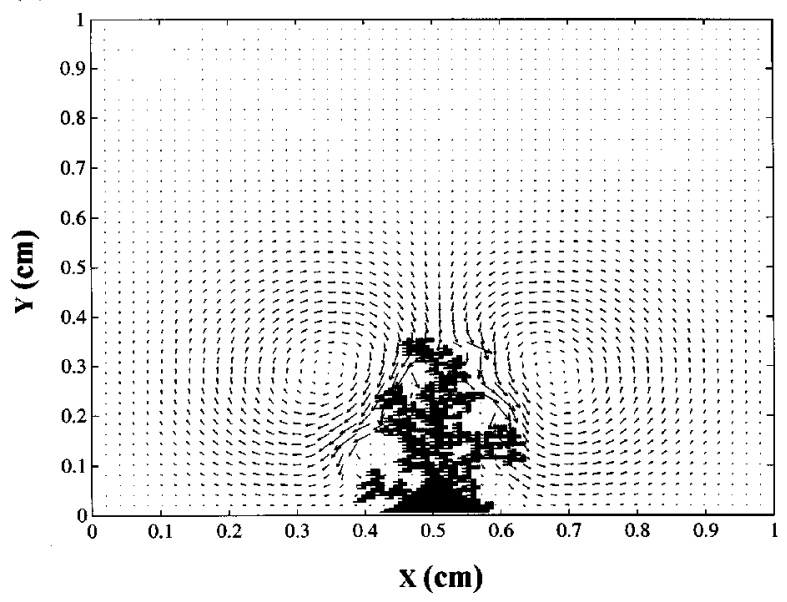

(b)

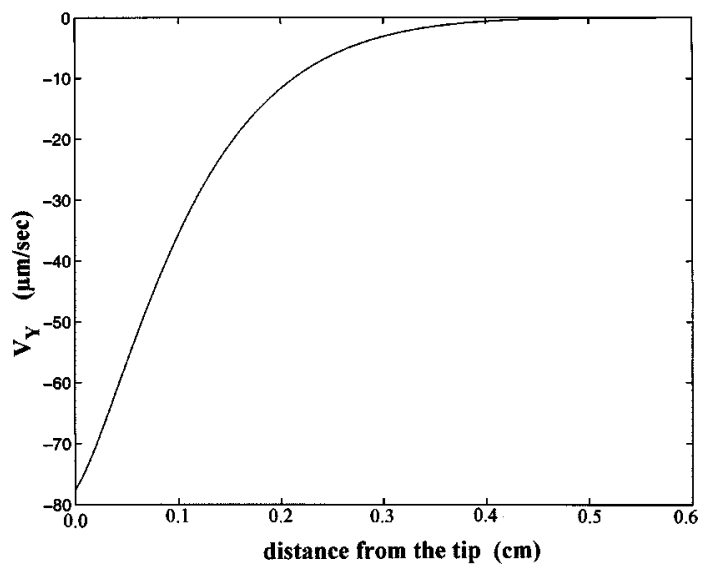

(c)

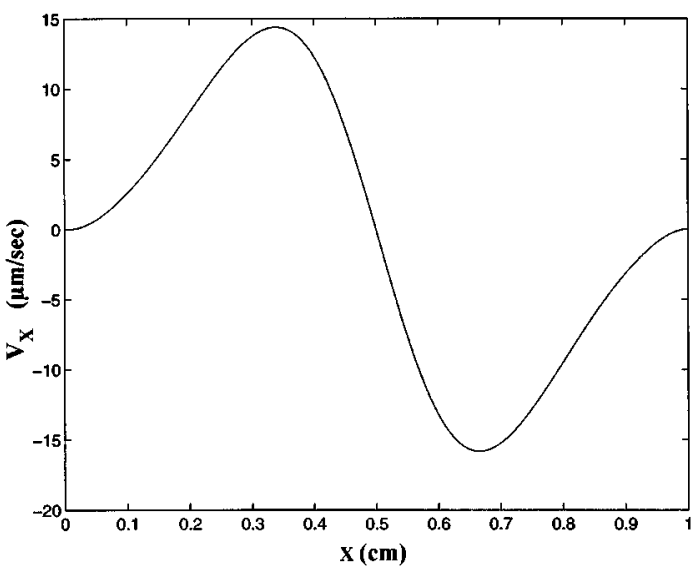

(d)

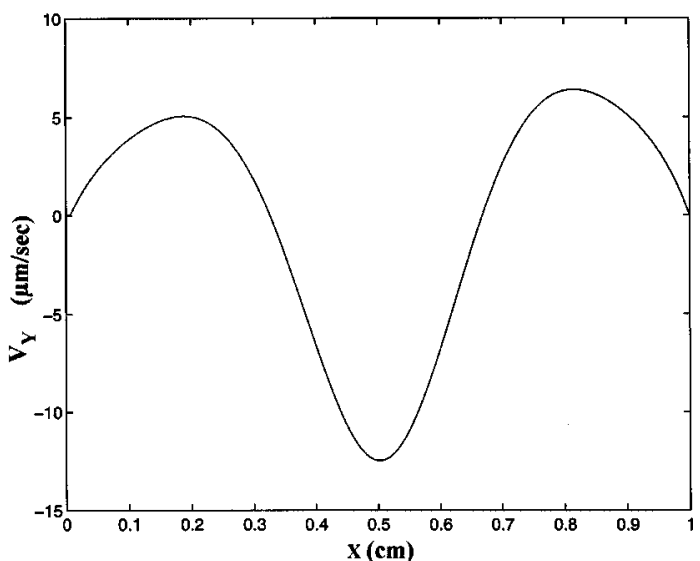

FIG. 8. (a) Simulation of the top view model showing convection around a growing tip (the cell is $1 \times 1 \mathrm{~cm}^{2}$ ) for Ge $=10^{3}$; $(\mathrm{b}) \mathrm{Velocity}$ component $V_{y}$ as a function of the distance to the tip along a vertical line passing through the tip (at $x=0.47 \mathrm{~cm}$ ). (c) Velocity component $V_{x}$ along a horizontal line close to the tip (at $y=0.6 \mathrm{~cm}$ ); (d) Velocity component $V_{y}$ along a horizontal line close to the tip (at $y$ $=0.6 \mathrm{~cm})$.

convection rolls in the simulations [Fig. 6(c)] is the same as that observed in experiments: the exponent is $4 / 5$ at early times and $1 / 2$ at long times.

Our simulations indicate that when buoyancy forces prevail, the gravity Grashof number Gg is the main dimensionless number governing the behavior; gravitoconvection increases with increasing $\mathrm{Gg}$. When electroconvection prevails, the model indicates that the Ge number governs the behavior; electroconvection increases for increasing Ge. The model also predicts the existence of vortex pairs formed by the electrical force on space charge accumulating near the growing filament tip [Fig. 8(a)]; similar vortex pairs are observed in experiment [Fig. 1(b)].

When ramified deposits form, they interact with the gravity current: the vortex tube bends and surrounds filaments, forming a sort of three-dimensional envelope tube squeezed by the filament tip and slaved to the deposit front. The inter- action among vortex rings due to Coulombic forces and vortex tubes due to buoyancy-driven forces in the presence of ramified deposits and their collision with the anode vortex tube is a complex, three-dimensional problem. A fuller understanding of this problem will require addressing the threedimensional character of ECD; hence detailed threedimensional measurements and simulations are needed.

\section{ACKNOWLEDGMENTS}

G.M. is thankful for the hospitality and generous support from the Center for Nonlinear Dynamics, University of Texas, Austin, during a visit on sabbatical from the University of Buenos Aires, and thanks F. Molina for helpful discussions. P.M. acknowledges support from the University of Buenos Aires. This work was partially supported by UBA Grant No. EX233/96, and by the U.S. Department of Energy Office of Basic Energy Sciences. 
[1] B. B. Mandelbrot, The Fractal Geometry of Nature (Freeman, San Francisco, 1982).

[2] T. Vicsek, Fractal Growth Phenomena, 2nd. ed. (World Scientific, Singapore, 1992).

[3] On Growth and Form, edited by G. Stanley and N. Ostrowsky Vol. 100 of NATO Advanced Study Institute Series B: Physics (Kluwer, Boston, 1986).

[4] L. Kadanoff, J. Stat. Phys. 39, 267 (1985).

[5] F. Argoul, J. Huth, P. Merzeau, A. Arneodo, and H. L. Swinney, Physica D 62, 170 (1993).

[6] R. M. Brady and R. C. Ball, Nature (London) 309, 225 (1984).

[7] D. Barkey, J. Electrochem. Soc. 138, 2912 (1991).

[8] V. Fleury, J. N. Chazalviel, M. Rosso, and B. Sapoval, Phys. Rev. A 44, 6693 (1991).

[9] V. Fleury, M. Rosso, and J. N. Chazalviel, Phys. Rev. A 43, 6908 (1991).

[10] V. Fleury, J. N. Chazalviel, and M. Rosso, Phys. Rev. Lett. 68, 2492 (1992).

[11] V. Fleury, J. N. Chazalviel, and M. Rosso, Phys. Rev. E 48, 1279 (1993).

[12] R. H. Cork, D. C. Pritchard, and W. Y. Tam, Phys. Rev. A 44, 6940 (1991).

[13] K. A. Linehan and J. R. de Bruyn, Can. J. Phys. 73, 177 (1995).

[14] V. Fleury, M. Rosso, and J. N. Chazalviel, in Ceramic Matrix Composites-Advanced High-Temperature Structured Materials, edited by R. A. Lowden, J. R. Hellmann, M. K. Ferber, S. G. DiPietro, and K. K. Chawla, MRS Symposia Proceedings No. 367 (Materials Research Society, Pittsburgh, 1995), p. 183.
[15] V. Fleury, J. Kaufman, and B. Hibbert, Phys. Rev. E 48, 3831 (1993).

[16] V. Fleury, J. Kaufman, and B. Hibbert, Nature (London) 367, 435 (1994).

[17] P. Carro, S. L. Marciano, A. Hernandez-Creus, S. Gonzalez, R.C. Salvarezza, and A. J. Arvia, Phys. Rev. E 48, 2374 (1993).

[18] J. Huth, H. L. Swinney, W. McCormick, A. Kuhn, and F. Argoul, Phys. Rev. E 51, 3444 (1995).

[19] D. Barkey, D. Watt, Z. Liu, and S. Raber, J. Electrochem. Soc. 141, 1206 (1994).

[20] C. Leger, J. Elezgaray, and F. Argoul, Phys. Rev. Lett. 78, 5010 (1997).

[21] A. J. Bard and L. R. Faulkner, Electrochemical Methods, Fundamentals and Applications (Wiley, New York, 1980).

[22] J. S. Newman, Electrochemical Systems (Prentice Hall, New Jersey, 1973).

[23] J. N. Chazalviel, Phys. Rev. A 42, 7355 (1990).

[24] G. Marshall, S. Tagtachian, and L. Lam, Chaos Solitons Fractals 6, 325 (1995).

[25] G. Marshall, E. Perone, P. Tarela, and P. Mocskos, Chaos Solitons Fractals 6, 315 (1995).

[26] G. Marshall, P. Mocskos, and M. Olivella, in Disordered Materials and Interfaces, edited by H. Z. Cummins, D. J. Durian, D. L. Johnson, and H. E. Stanley, MRS Symposia Proceedings No. 407 (Materials Research Society, Pittsburgh, 1996), p. 355.

[27] G. Marshall and P. Mocskos, Phys. Rev. E 55, 549 (1997).

[28] J. N. Chazalviel, M. Rosso, E. Chassaing, and V. Fleury, J. Electroanal. Chem. 407, 61 (1996).

[29] V. G. Levich, Physicochemical Hydrodynamics (Prentice Hall, New Jersey, 1962). 